\title{
Effects of 14 Weeks Yoga Training on Labor Pain and Pregnancy Outcomes in Primiparous Women
}

\section{ART ICLE INF O}

\section{Article Type}

Original Research

\section{Authors}

Gholipour Sh. ${ }^{* 1} M S c$

Memari E. ${ }^{2} M S C$

Shojaedin S.S. ${ }^{3} P h D$

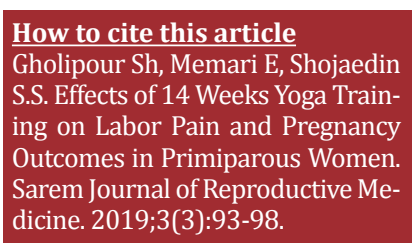

${ }^{1}$ Exercise Physiology Department, North Tehran Branch, Islamic Azad University, Tehran, Iran

${ }^{2}$ Sarem Fertility and Infertility Research Center (SAFIR), Sarem Women's Hospital, Tehran, Iran ${ }^{3}$ Exercise Physiology Department, Physical Education \& Sport Sciences Faculty, Kharazmi University, Tehran, Iran

\section{*Correspondence}

Address: Exercise Physiology Department, North Tehran Branch, Islamic Azad University, Tehran, Iran Phone: -

Fax: -

shadigholipour184@yahoo.com

\section{Article History}

Received: February 24, 2018

Accepted: March 6, 2019

ePublished: July 6, 2019

\section{A B S T R A C T}

Aims The experience of labor pain is a complicated, individual, and multiple response to the sensory stimulation produced during birth. The aim of this study was to investigate the effects of yoga training after 14 weeks on labor pain and pregnancy outcomes in primiparous women. Materials \& Methods In this clinical trial, 80 primiparous pregnant women aged 25-35 years old referred to Sarem Hospital in Tehran, in 2017, were selected by purposive sampling method and randomly divided into experimental $(n=40)$ and control $(n=40)$ groups. The experimental group started practicing yoga from the 14th week of pregnancy, but the control group did not receive any intervention. The severity of labor pain and Apgar scores were compared. The data were analyzed by SPSS 23 software using one-way analysis of variance, Tukey post hoc test, and independent t-test.

Findings Yoga training reduced the severity of labor pain in primiparous women $(F=38.57$; $\mathrm{p}<0.001)$. Also, yoga exercises had a significant effect on the first- and fifth-minute Apgar scores $(\mathrm{F}=10.64 ; \mathrm{p}<0.001)$. In addition, yoga training had a significant effect on neonatal weight $(\mathrm{t}=5.123 ; \mathrm{p}<0.001)$.

Conclusion Yoga training reduces the severity of labor pain in primiparous women and has beneficial effects for pregnant women and their infants.

Keywords Yoga; Severity of Labor Pain; Primiparity; Pregnancy

\section{CITATION L INKS}

[1] Has the medicalisation of childbirth ... [2] Duration of the second stage of labor in multiparous ... [3] Exercise training in pregnancy reduces offspring size without changes ... [4] Effects of aerobic exercise training on maternal and neonatal outcome ... [5] Efficacy of antenatal exercises on maternal and neonatal outcomes ... [6] The impact of exercise during pregnancy on neonatal outcomes: A randomized ... [7] Effect of physical activity during pregnancy on birth outcomes ... [8] Factors related to exercise over the course of pregnancy including women's beliefs ... [9] Danforth's obstetrics and ... [10] Does exercise training during pregnancy affect gestational ... [11] Investigation of effects of aerobic exercise on pregnancy and its ... [12] Aerobic exercise for women during ... [13] Treatment of pregnancyrelated lumbar and pelvic girdle pain by the yoga method: A randomized ... [14] Effects of a prenatal yoga programme on the discomforts of pregnancy and maternal childbirth selfefficacy in ... [15] The prevalence and characteristics of young and mid-age women who use yoga and meditation: Results of a nationally representative survey of ... [16] Yoga during pregnancy ... [17] Exercise in pregnancy: Effect on fitness and obstetric outcomes-a ... [18] Water aerobics II: Maternal body composition and perinatal outcomes after a program for low risk pregnant ... [19] Committee on Obstetric Practice American Academy of Pediatrics - Committee on Fetus and ... [20] Apgar scores: Examining the long-term ... [21] Effect of the integrated approach of yoga therapy on platelet count and uric ... [22] Assessment of effects of Kegel exercises on reduction of perineal ... [23] The effect of training respiratory exercises on the severity of labor pain in ... [24] Yoga practice for the management of type II diabetes mellitus in adults: A ... [25] The health benefits of yoga and exercise: A review of ... [26] Physical exercise during pregnancy and the risk of preterm birth: A study ... [27] Leisure time physical activity is associated with a reduced ... [28] The effects of breathing patterns and massage on the pain and ... [29] Effect of physical exercise on pregnancy ... [30] The effect of an aerobic exercise course on ... [31] Comparison of the outcome of pregnancy and infant health at birth in mothers of athletes and ... [32] The effect of vigorous exercise during pregnancy ... [33] Maternal and fetal responses to a maternal ... 


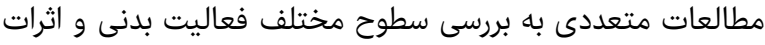

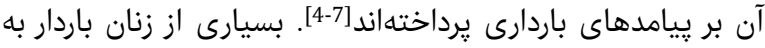

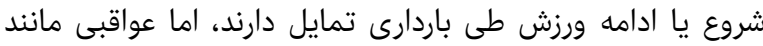

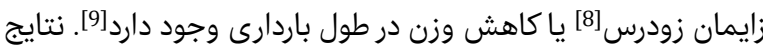

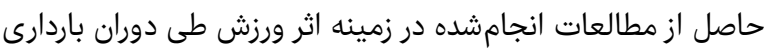

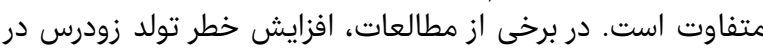

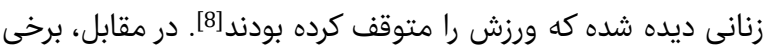

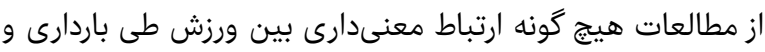

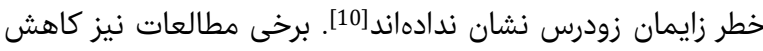

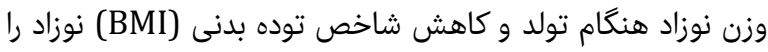

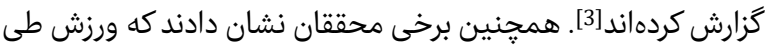

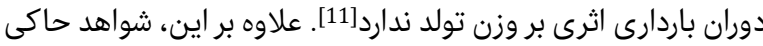

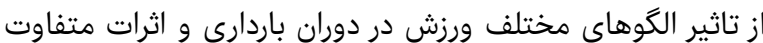

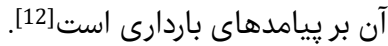

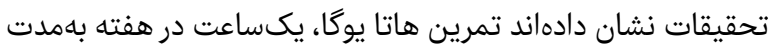

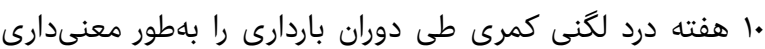

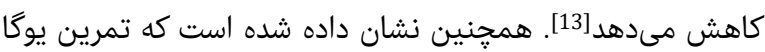

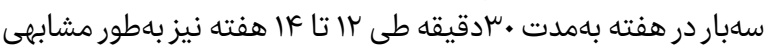

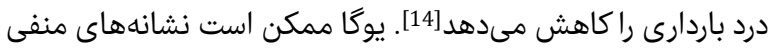

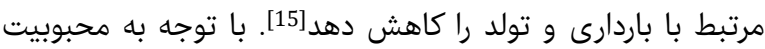

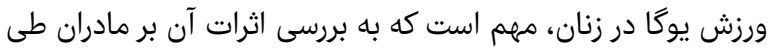

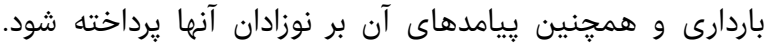

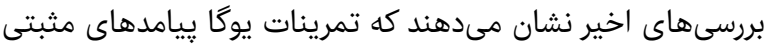

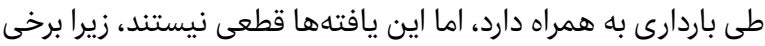

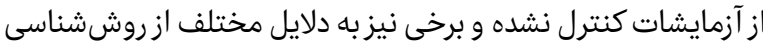

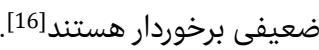

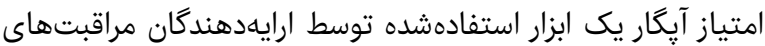

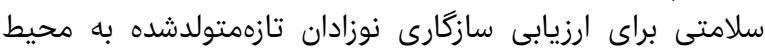

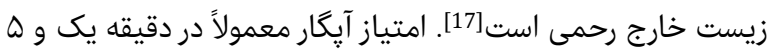

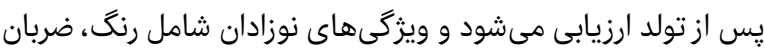

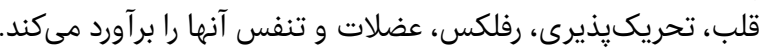

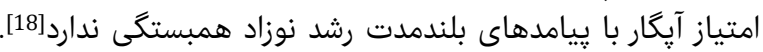

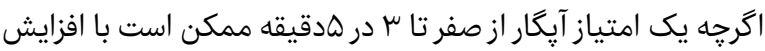

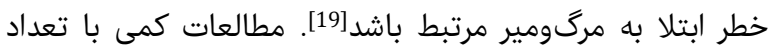

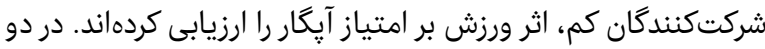

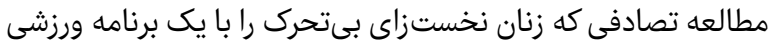

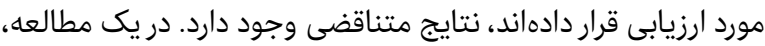

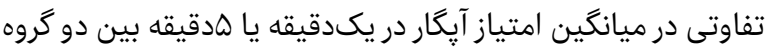

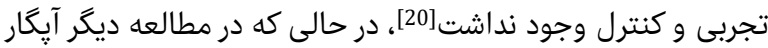

يك و هدقيقه در گروه ورزش بالاتر بود [6]

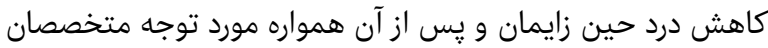

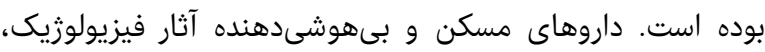

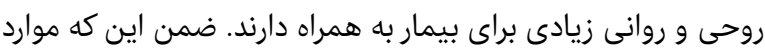

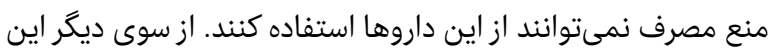

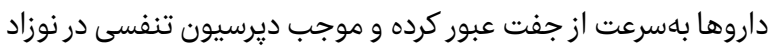

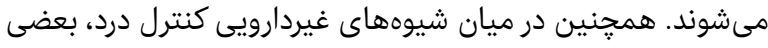

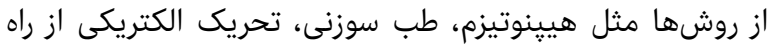

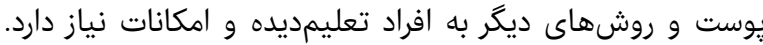

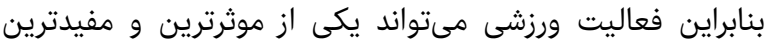

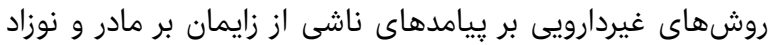

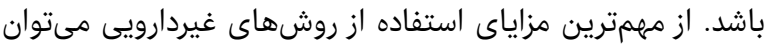

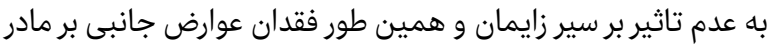

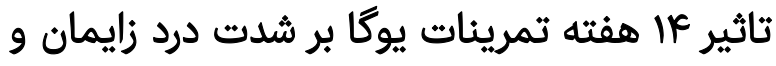

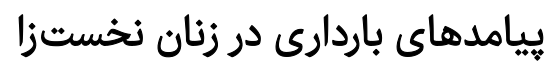

MSc " Mادى قلىيور

كروه فيزيولوزى ورزشى، واحد تهريوران شمال، دانشگاه آزاد اسلامى، تهران، ايران

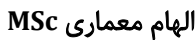

مركز تحقيقات بارورى و نابارورى صارم، بيمارستان صارم، تهران، ايران

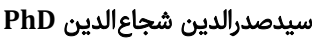

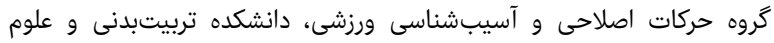
ورزشى، دانشكاه خوارزمى، تهران، ايران آسيني

جكيده

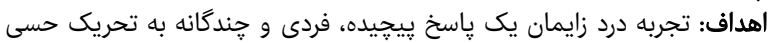

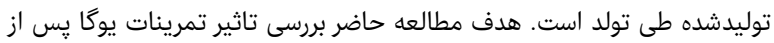

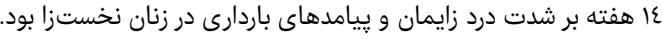

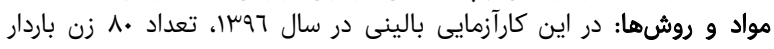

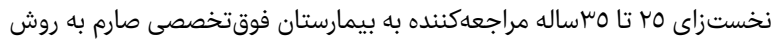

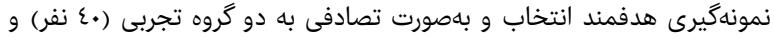

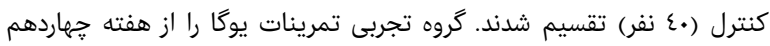

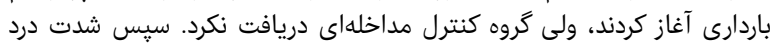

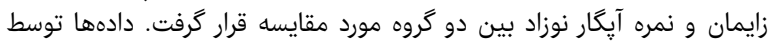

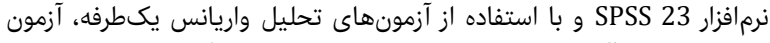

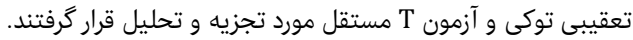

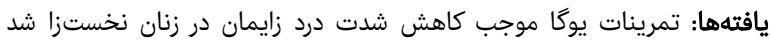

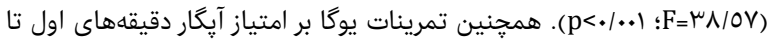

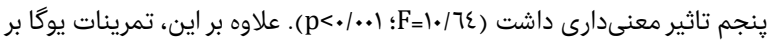

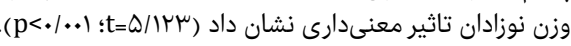

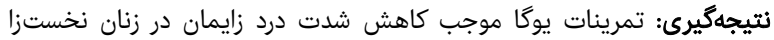

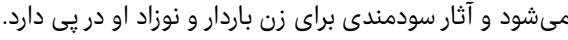

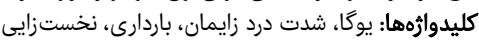

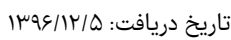

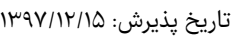

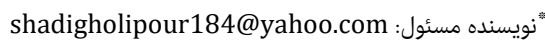

مقدمه - مقد

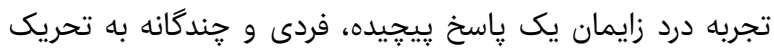

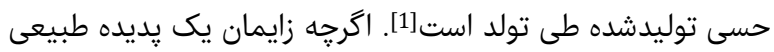

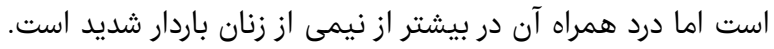

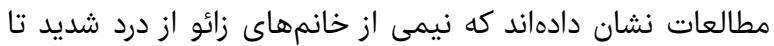

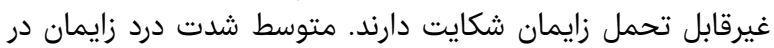

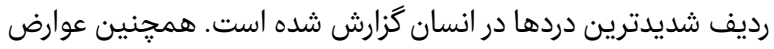

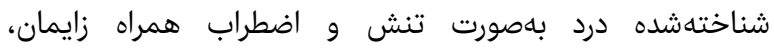

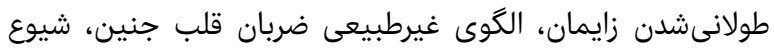

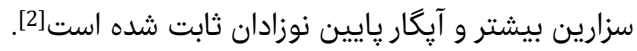

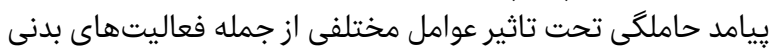

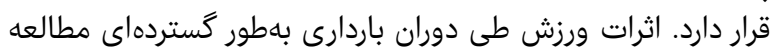

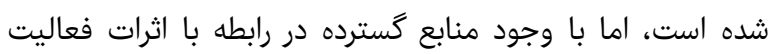

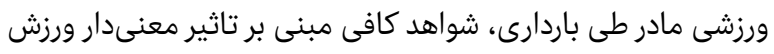

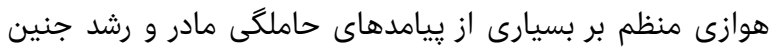

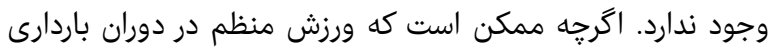

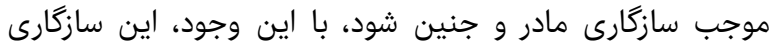

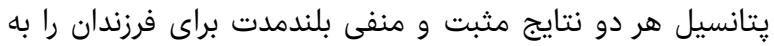

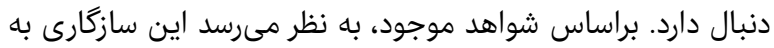

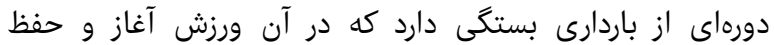
مى شود[3]. 


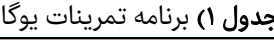

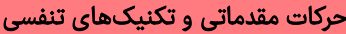

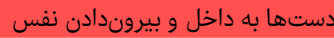

\begin{tabular}{|c|c|}
\hline & حركات مقدماتى و تكنيكهاى تنفسى \\
\hline 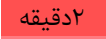 & دستها به داخل و بيروندادن نفس \\
\hline 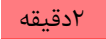 & دستها در حالت كشيده و نفس آرام \\
\hline يكدقيقه & زانوها در حالت كشيده و نفسكشيدن با حمايت ديوار \\
\hline 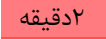 & نفسكشيدن همراه با حرخش به اطراف بدن \\
\hline 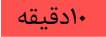 & ريلكسيشن عميق \\
\hline 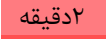 & نفسكشيدن همرا با بالابردن ياها \\
\hline r ادقيقه & نفسكشيدن همرا با بالابردن مفصل ران \\
\hline r ادقيقه & انجام حركت يا دوجرخه در وضعيت خوابيده به يشت \\
\hline 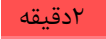 & وضعيت خوابيده روى شكم، كشش و تنفس آرام \\
\hline 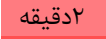 & تنفس آرام همراه با حركت كشش گربه \\
\hline هدقيقه & ريلكسيشن عميق \\
\hline 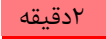 & קرخش زانو \\
\hline & تكنيكهاى ريلكسيشن (آرامسازى) \\
\hline 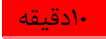 & ريلكسيشن عميق \\
\hline & هراناياما \\
\hline r ادقيقه & تنفس متناوب با بينى \\
\hline 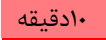 & شاوآسانا جانبى \\
\hline & تكنيكهاى مديتيشن \\
\hline r ادقيقه & تمرينات جشمها \\
\hline · سرقيقه & نكنيك تصويرسازى ذهنى، مجسمسازى هدايتشده و \\
\hline
\end{tabular}

يافتهها

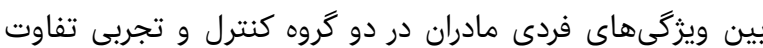

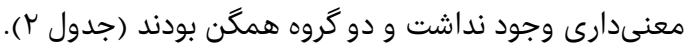

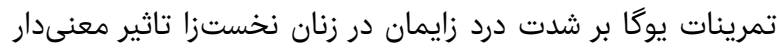

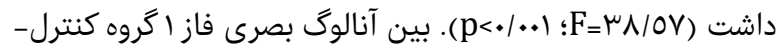

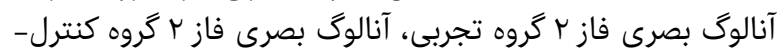

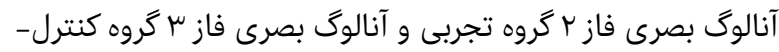

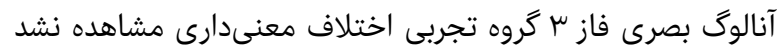

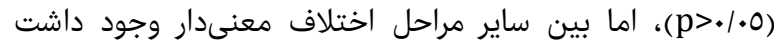

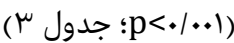

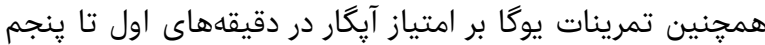

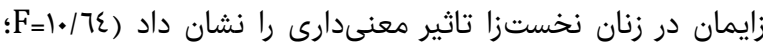

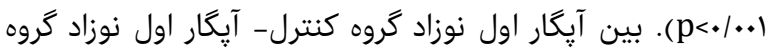

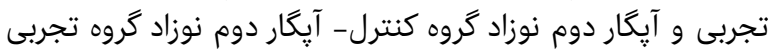

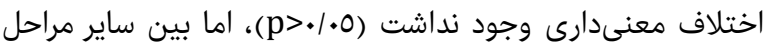

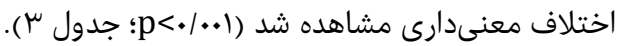

جدول r) ميانگين آمارى مربوط به ويزگى هاى فردى مادران در دو گروه كنترل و

تجربى

\begin{tabular}{|c|c|c|}
\hline كروه تجربى & كروه كنترل & متغيرها \\
\hline$r q / \mu \pm \varepsilon \cdot 1 \cdot 1$ & $\mu \cdot / \gamma \pm V \cdot / q \mu$ & سن (سال) \\
\hline$\left|\varsigma \psi / \kappa_{ \pm} \cdot \Delta / \varphi\right|$ & $\mid \& K / \Delta \pm K \Delta / T$. & قد (سانتىمتر) \\
\hline$G V / \mathcal{F} \pm V \Delta / 19$ & $Q q / K \pm F Q / \wedge q$ & وزن (كيلوگرم) \\
\hline$\Psi \Lambda / I \pm \mid Q / 1 Q$ & $\mu \vee / I \pm q \Delta / \mu l$ & سن حاملكى (هفته) \\
\hline
\end{tabular}

جدول سا) ميانگين آمارى مربوط به وزن نوزاد و نمرات آيكار اول و دوم نوزادان و

\begin{tabular}{|c|c|c|}
\hline كروه تجربى & كروه كنترل & متغيرها \\
\hline$r \Delta \Delta \cdot / K \mid q \pm r \Delta / A K$ & $r q 99 / r \wedge k \pm \Delta \cdot / \cdot V$ & وزن نوزاد (كرم) \\
\hline & & يخار نوزاد \\
\hline$V / I \pm r \cdot / k V$ & $V / I \pm K \cdot / r V$ & ول \\
\hline$\Lambda / \pm \pm q \Delta / \mu q$ & 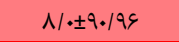 & دوم \\
\hline & & نالوى بصرى درد \\
\hline$\kappa / l \pm \cdot \Delta / F \varepsilon$ & $\Delta / 1 \pm \Delta \cdot / \vee \cdot$ & غاز 1 \\
\hline$G / I \pm V \cdot / F \Delta$ & $V / I \pm I . / \mu \mu$ & كاز r \\
\hline$\Lambda / / \pm V Q / \Delta I$ & $q / / \pm F \cdot / \cdot k$ & 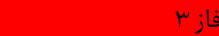 \\
\hline
\end{tabular}

و جنين اشاره كرد. با توجه به سياست كشورى مبنى بر كاهش آمار

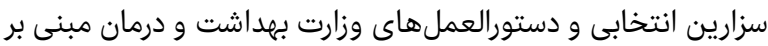

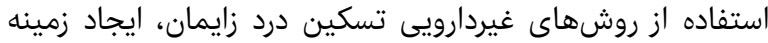

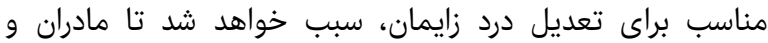

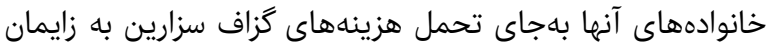

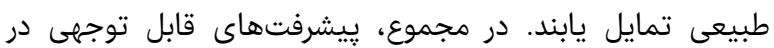

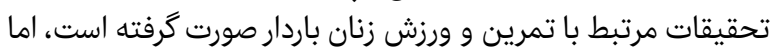

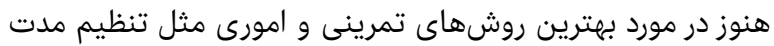

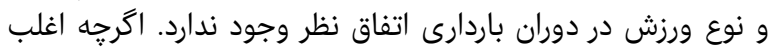

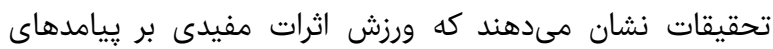

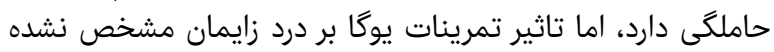

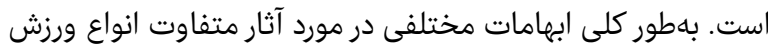

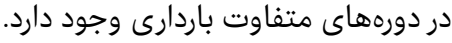

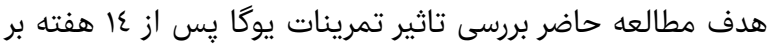

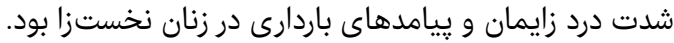

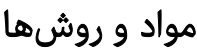

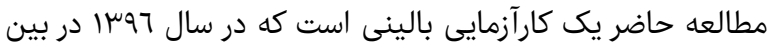

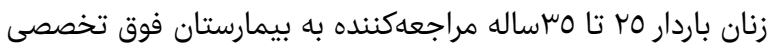

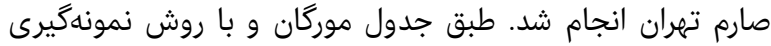

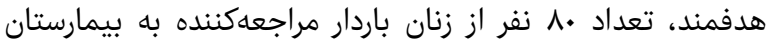

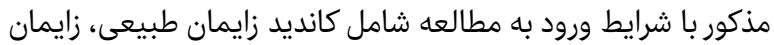

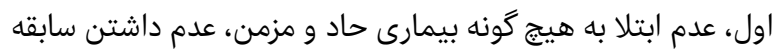

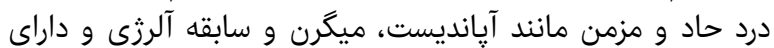

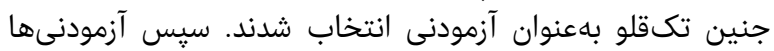

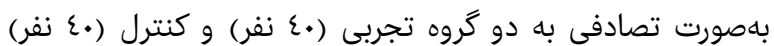

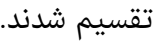
مادران باردار مراجعهكننده به بيمارستان فوق تخصصى صارم تهران

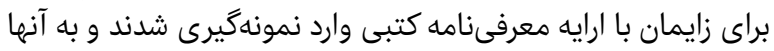

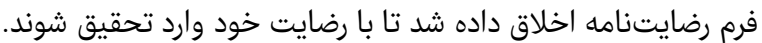

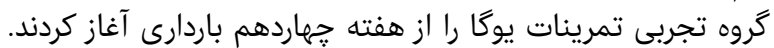

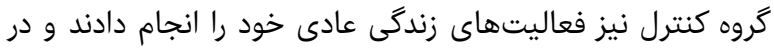

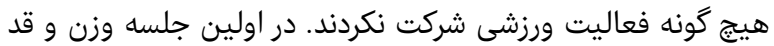

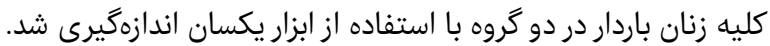

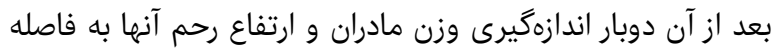

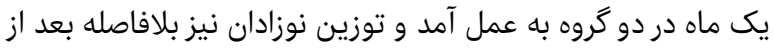

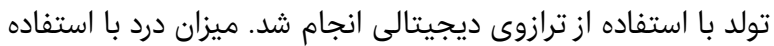

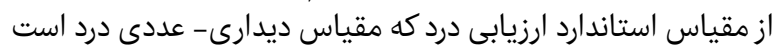

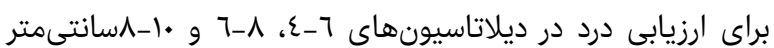

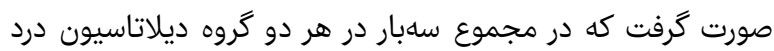

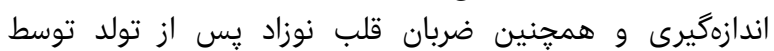

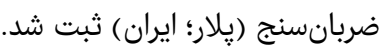

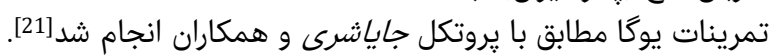

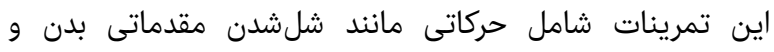

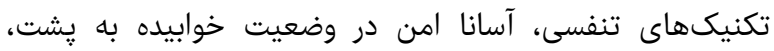

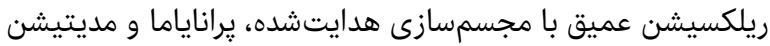

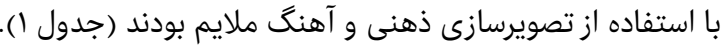

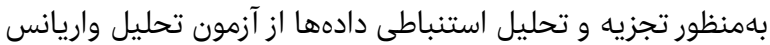

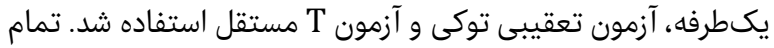

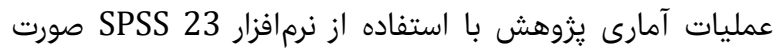

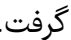


اكثر نوزادان در هر دو كروه داراى ميانكين امتياز آيكار 9 بودند.

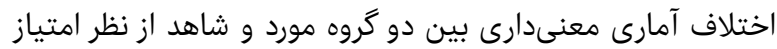

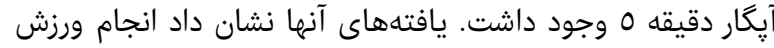

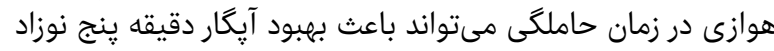

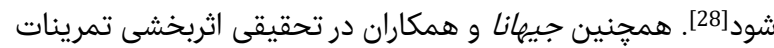

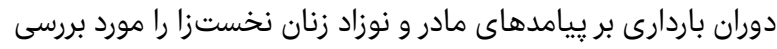

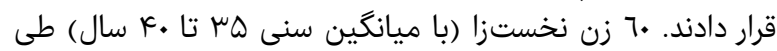

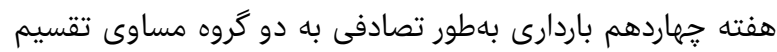

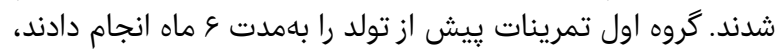

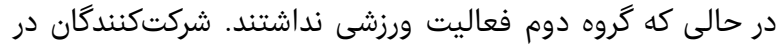

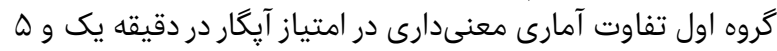

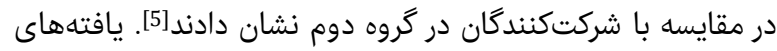

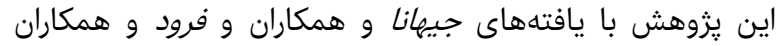

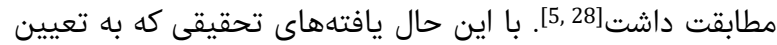

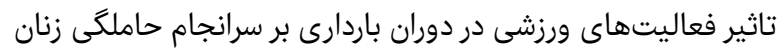

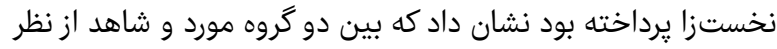

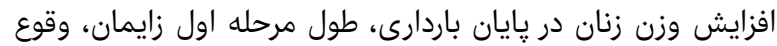

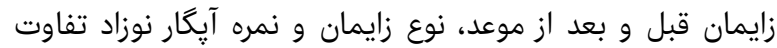

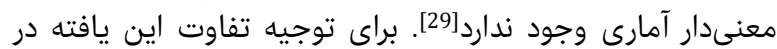

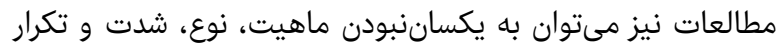

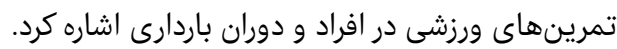

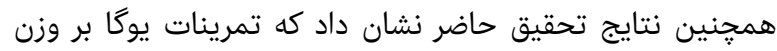

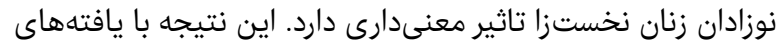

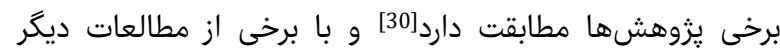

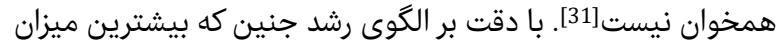

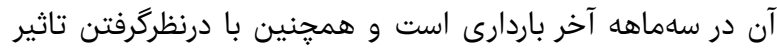

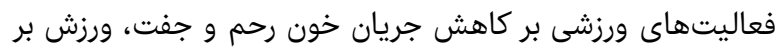

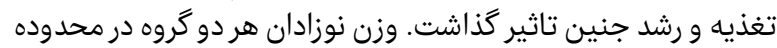

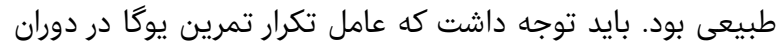

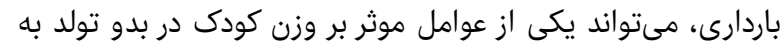

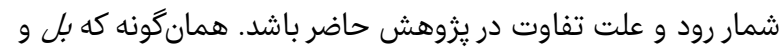

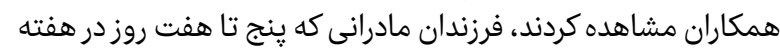

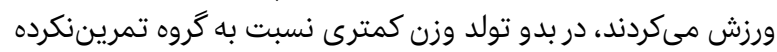

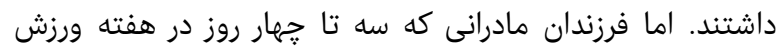

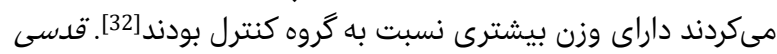

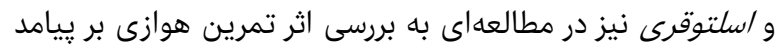

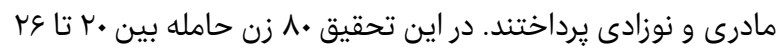

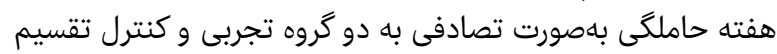

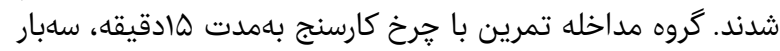

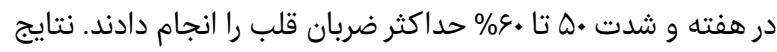

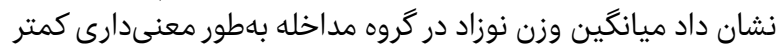

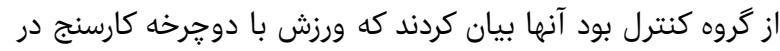

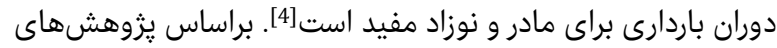

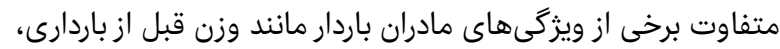

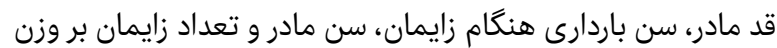

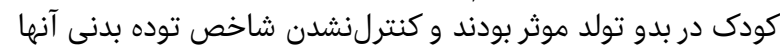

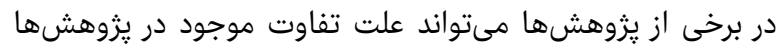

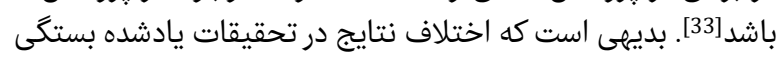

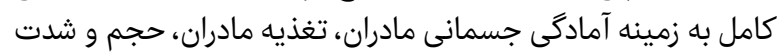

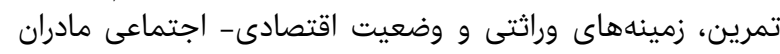

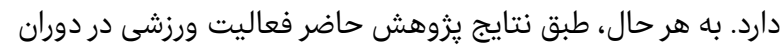

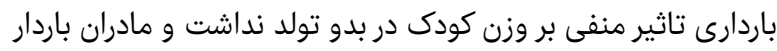

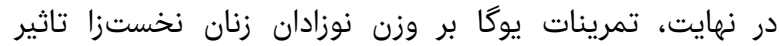

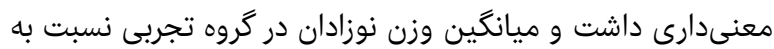

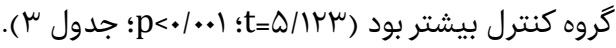

بحث مثرف

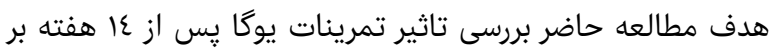

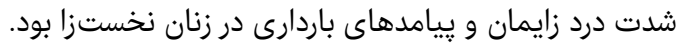

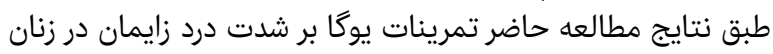

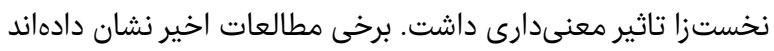

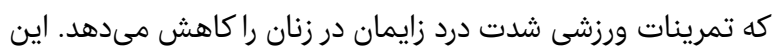

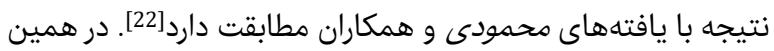

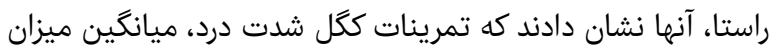

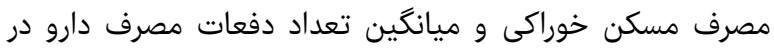

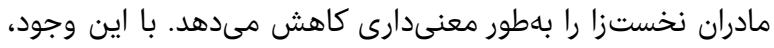

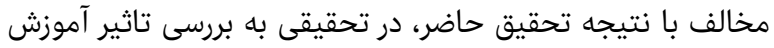

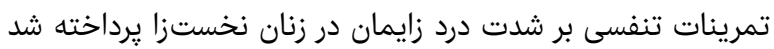

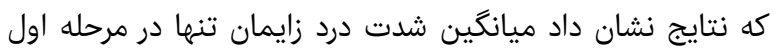

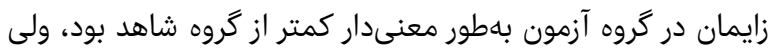

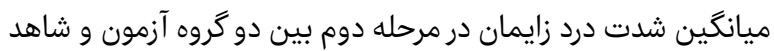

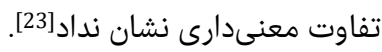

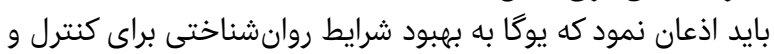

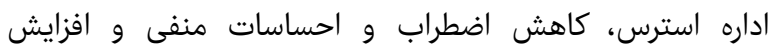

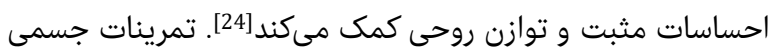

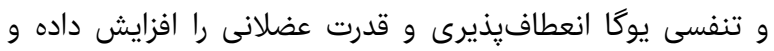

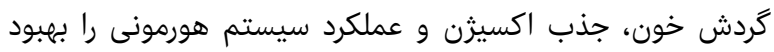

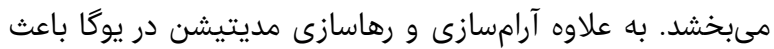

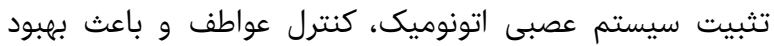

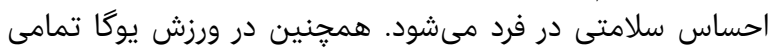

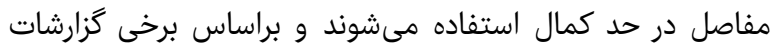

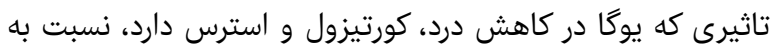

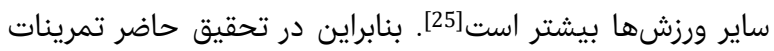

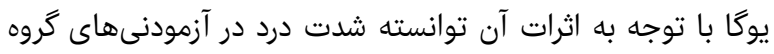

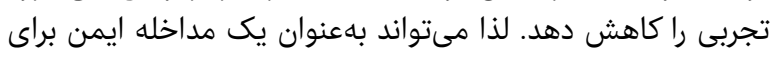

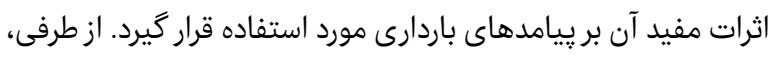

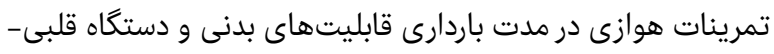

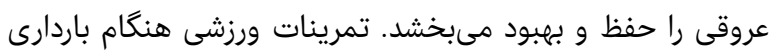

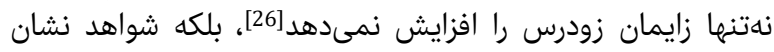

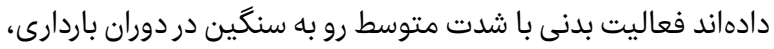

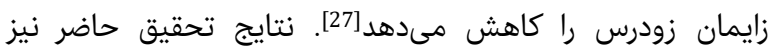

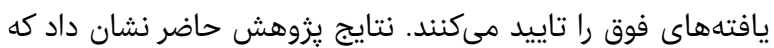

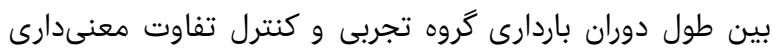

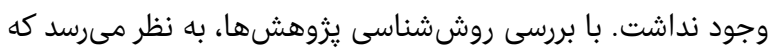

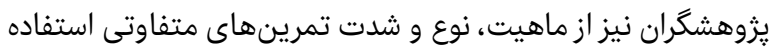

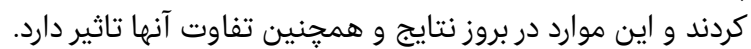

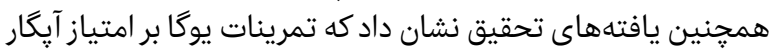

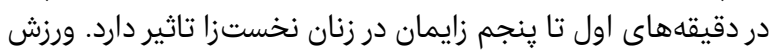

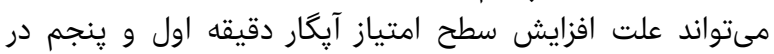

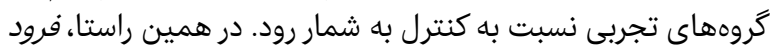

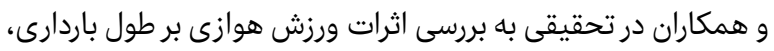

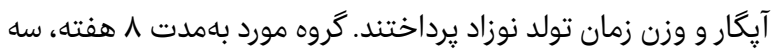

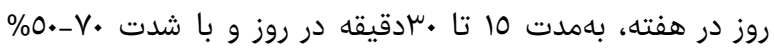
حداكثر ظرفيت هوازى (VO) 


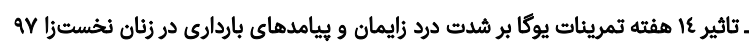

9- Gibbs RS, Karlan BY, Haney AF, Nygaard IE. Danforth's obstetrics and gynecology. $10^{\text {th }}$ Edition. Philadelphia PA: Lippincott Williams \& Wilkins; 2008.

10- Barakat R, Stirling JR, Lucia A. Does exercise training during pregnancy affect gestational age? a randomised controlled trial. Br J Sports Med. 2008;42(8):674-8.

11- Memari A, Ramim T, Amini M, Mehran A, Ajorlu A, Shakibaee P. Investigation of effects of aerobic exercise on pregnancy and its circumstances. Hayat. 2006;12(3):3541. [Persian]

12- Kramer MS, Mc Donald SW. Aerobic exercise for women during pregnancy. Cochrane Database Syst Rev. 2006;(3):CD000180.

13- Martins RF, Pinto e Silva JL. Treatment of pregnancyrelated lumbar and pelvic girdle pain by the yoga method: A randomized controlled study. J Altern Complement Med. 2014;20(1):24-31.

14- Sun YC, Hung YC, Chang Y, Kuo SC. Effects of a prenatal yoga programme on the discomforts of pregnancy and maternal childbirth self-efficacy in Taiwan. Midwifery. 2010;26(6):e31-6.

15- Sibbritt D, Adams J, Van Der Riet P. The prevalence and characteristics of young and mid-age women who use yoga and meditation: Results of a nationally representative survey of 19,209 Australian women. Complement Ther Med. 2011;19(2):71-7.

16- Babbar S, Parks-Savage AC, Chauhan SP. Yoga during pregnancy: A review. Am J Perinatol. 2012;29(6):459-64. 17- Price BB, Amini SB, Kappeler K. Exercise in pregnancy: Effect on fitness and obstetric outcomes-a randomized trial. Med Sci Sports Exerc. 2012;44(12):2263-9.

18- Cavalcante SR, Cecatti JG, Pereira RI, Baciuk EP, Bernardo AL, Silveira C. Water aerobics II: Maternal body composition and perinatal outcomes after a program for low risk pregnant women. Reprod Health. 2009;6:1.

19- Committee on Obstetric Practice American Academy of Pediatrics - Committee on Fetus and Newborn. Committee opinion No. 644: The Apgar score. Obstet Gynecol. 2015;126(4):e52-5.

20- Montgomery KS. Apgar scores: Examining the longterm significance. J Perinat Educ. 2000;9(3):5-9.

21- Jayashree R, Malini A, Rakhshani A, Nagendra $H_{\text {, }}$ Gunasheela S, Nagarathna R. Effect of the integrated approach of yoga therapy on platelet count and uric acid in pregnancy: A multicenter stratified randomized singleblind study. Int J Yoga. 2013;6(1):39-46.

22- Mahmoodi F, Mobaraki A. Assessment of effects of Kegel exercises on reduction of perineal pain after episiotomy in primiparous women. Iran J Obstet Gynecol Infertil. 2014;17(95):18-25. [Persian]

23- Hasanpour Azghadi SB, Salari E. The effect of training respiratory exercises on the severity of labor pain in primiparous women. Daneshvar Med. 2006;13(63):9-14. [Persian]

24- Aljasir B, Bryson M, Al-Shehri B. Yoga practice for the management of type II diabetes mellitus in adults: A systematic review. Evid Based Complement Alternat Med. 2010;7(4):399-408.

25- Ross A, Thomas S. The health benefits of yoga and exercise: A review of comparison studies. J Altern Complement Med. 2010;16(1):3-12.

26- Juhl M, Andersen PK, Olsen J, Madsen M, Jørgensen T, Nøhr EA, et al. Physical exercise during pregnancy and the risk of preterm birth: A study within the Danish National Birth Cohort. Am J Epidemiol. 2008;167(7):859-66.

27- Hegaard HK, Hedegaard M, Damm P, Ottesen B, Petersson K, Henriksen TB. Leisure time physical activity
سالم توانستند با نيازهاى فيزيولوزيك فعاليت ورزشى و رشد كودى سازگارى ييدا كنند.

با توجه به مطالعات اندك انجامشده ديد در اين رابطه، تاييد ارتباط بين

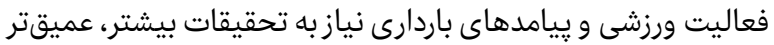

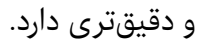

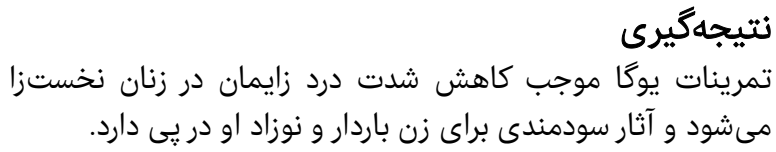

تشكر و قدردانى: بدينوسيله از مشاركت و همراهى تمامى بانوان باردار كه در اين يزوهش شرداني بدركت كرده و محققان را يارى نمودند كمال

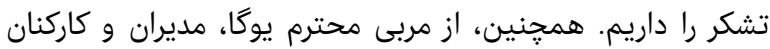

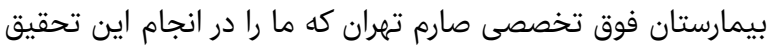

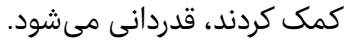
تاييديهاخلاقى: مطالعه حاضر توسط كميته اخلاق درد يزون يزوهشعاه علوم ورزشى با كد

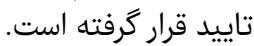

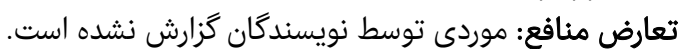

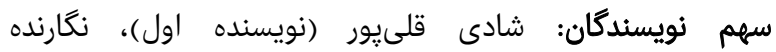

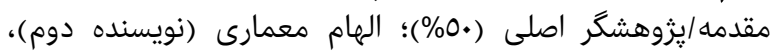

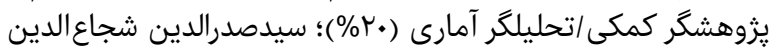

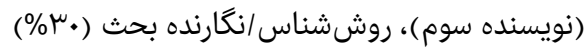

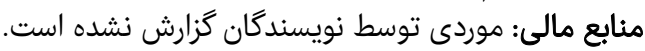

\section{منابع}

1- Johanson R, Newburn M, Macfarlane A. Has the medicalisation of childbirth gone too far?. BMJ. 2002;324(7342):892-5.

2- Cheng YW, Hopkins LM, Laros RK Jr, Caughey AB. Duration of the second stage of labor in multiparous women: Maternal and neonatal outcomes. Am J Obstet Gynecol. 2007;196(6):585.

3- Hopkins SA, Baldi JC, Cutfield WS, Mc Cowan L, Hofman PL. Exercise training in pregnancy reduces offspring size without changes in maternal insulin sensitivity. J Clin Endocrinol Metab. 2010;95(5):2080-8.

4- Ghodsi Z, Asltoghiri M. Effects of aerobic exercise training on maternal and neonatal outcome: A randomized controlled trial on pregnant women in Iran. J Pak Med Assoc. 2014;64(9):1053-6.

5- Gehan AA, Khadiga SA, Amir GA, Eman A. Efficacy of antenatal exercises on maternal and neonatal outcomes in elderly primigravida. Kasr Al Ainy Med J. 2015;21(3):10914.

6- Murtezani A, Paçarada M, Ibraimi Z, Nevzati A, Abazi N. The impact of exercise during pregnancy on neonatal outcomes: A randomized controlled trial. J Sports Med Phys Fitness. 2014;54(6):802-8.

7- Jayakody O, Senanayake H. Effect of physical activity during pregnancy on birth outcomes in mothers presenting at the antenatal clinic of De Soysa Maternity Hospital, Colombo 08. Asia Pac J Multidiscip Res. 2015;3(2):76-82.

8- Duncombe D, Wertheim EH, Skouteris H, Paxton SJ, Kelly L. Factors related to exercise over the course of pregnancy including women's beliefs about the safety of exercise during pregnancy. Midwifery. 2009;25(4):430-8. 
Olympic. 2006;14(2):63-72. [Persian]

31- Rahmaninia F, Mohebbi H, Sam F. Comparison of the outcome of pregnancy and infant health at birth in mothers of athletes and non-athletes. Harakat. 2003;(16):39-56. [Persian]

32- Bell RJ, Palma SM, Lumley JM. The effect of vigorous exercise during pregnancy on birth-weight. Aust N Z J Obstet Gynaecol. 1995;35(1):46-51.

33- Collings CA, Curet LB, Mullin JP. Maternal and fetal responses to a maternal aerobic exercise program. Am J Obstet Gynecol. 1983;145(6):702-7.

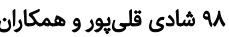
is associated with a reduced risk of preterm delivery. Am J Obstet Gynecol. 2008;198(2):180.

28- Foroud A, Foroud A, Mehdipour S. The effects of breathing patterns and massage on the pain and perception of labor in primiparous women. J Shahrekord Univ Med Sci. 2006;7(4):70-7. [Persian]

29- Hajikazemi E, Heydari M, Feizi Z, Haghani H. Effect of physical exercise on pregnancy outcome. Iran J Nurs. 2000;13(25):15-22. [Persian]

30- Sedaghati P, Agha Alinejad H, Arjmand A. The effect of an aerobic exercise course on the outcome of pregnancy. 